\title{
MOTIVACIÓN: PERSPECTIVAS TEÓRICAS Y ALGUNAS CONSIDERACIONES DE SU IMPORTANCIA EN EL ÁMBITO EDUCATIVO
}

\author{
María Luisa Naranjo Pereira \\ Docente de Escuela de Orientación y Educación Especial \\ Universidad de Costa Rica \\ San José, Costa Rica
}

Recibido 27-VI-2009 • Aceptado 21-VIII-2009 • Corregido 5-X-2009

\begin{abstract}
Resumen: Este artículo tiene como propósito revisar el concepto de motivación, su evolución histórica, los tres principales enfoques relacionados con esta, a saber, conductual, humanista y cognitivo; y, además, presentar algunos aspectos de importancia sobre la motivación en el ámbito educativo. El enfoque conductual enfatiza que las personas actúan motivadas por beneficios o recompensas que puedan recibir. El humanista subraya las cualidades del ser humano, su capacidad para desarrollarse y su libertad de elección. De este enfoque se estudian las teorías motivacionales: Jerarquía de las necesidades de Maslow, Existencia, relación y crecimiento de Alderfer y la Teoría de las necesidades de McClelland. El enfoque cognitivo señala que lo que se piensa que puede ocurrir determina lo que sucede; así, las percepciones de los eventos influyen sobre las emociones y los comportamientos. Si la persona cree que fracasará es probable que fracase y si piensa que tendrá éxito posiblemente lo logre. En esta perspectiva, los pensamientos guían la motivación. De este enfoque se revisa la Teoría de las expectativas de Vroom, la Teoría de la equidad de Adams y el Modelo de fijación de metas y objetivos al que se refiere Trechera. Sobre el ámbito educativo se analiza especialmente la relación entre emoción y motivación y la relación entre la Teoría de las atribuciones y el desempeño académico. Se presentan algunos motivos que pueden orientar el comportamiento estudiantil $y$, finalmente, se estudian las motivaciones intrínseca y extrínseca, y su influencia en el rendimiento académico del estudiantado.
\end{abstract}

Palabras clave: Enfoques, motivación, educación.

\section{Introducción: El concepto de motivación}

La motivación es un aspecto de enorme relevancia en las diversas áreas de la vida, entre ellas la educativa y la laboral, por cuanto orienta las acciones y se conforma así en un elemento central que conduce lo que la persona realiza y hacia qué objetivos se dirige. De acuerdo con Santrock (2002), la motivación es "el conjunto de razones por las que las personas se comportan de las formas en que lo hacen. El comportamiento motivado es vigoroso, dirigido y sostenido" (p. 432).

Ajello (2003) señala que la motivación debe ser entendida como la trama que sostiene el desarrollo de aquellas actividades que son significativas para la persona y en las que esta toma parte. En el plano educativo, la motivación debe ser considerada como la disposición positiva para aprender y continuar haciéndolo de una forma autónoma.

Trechera (2005) explica que, etimológicamente, el término motivación 


\begin{abstract}
This article has for objective to review the concept of motivation, its historical development, and the three main approaches about it: behavioral, cognitive and humanistic, and some important aspects of the motivation on the educational ambit. The first approach emphasizes that people are motivated by the profit or reward they can receive. The humanistic underlines the qualities of human beings, their ability to grow and their freedom of choice. This approach looks at the motivational theories: Hierarchy of Needs by Maslow, Existence, Relation and Growth Theory by Alderfer and McClelland's Theory of Needs. The cognitive approach points out that the thought we think might happen determines what happens. Thoughts guide motivation. From this approach we review the Expectations Theory of Vroom, the Equity Theory Adams and the Model Setting Goals and Objectives from Trechera. Especially in the field of education examines the relationship between emotion and motivation, and the relationship between the Theory of Attributions and academic performance. Are some reasons that can guide student behavior and finally explores the intrinsic and extrinsic motivation and its influence in the academic performance of the students.
\end{abstract}

Key words: Approach, motivation, education. procede del latín motus, que se relaciona con aquello que moviliza a la persona para ejecutar una actividad. De esta manera, se puede definir la motivación como el proceso por el cual el sujeto se plantea un objetivo, utiliza los recursos adecuados y mantiene una determinada conducta, con el propósito de lograr una meta. Según Bisquerra (2000):

La motivación es un constructo teórico-hipotético que designa un proceso complejo que causa la conducta. En la motivación intervienen múltiples variables (biológicas y adquiridas) que influyen en la activación, direccionalidad, intensidad y coordinación del comportamiento encaminado a lograr determinadas metas (p. 165).

Herrera, Ramírez, Roa y Herrera (2004) indican que la motivación es una de las claves explicativas más importantes de la conducta humana con respecto al porqué del comportamiento. Es decir, la motivación representa lo que originariamente determina que la persona inicie una acción (activación), se dirija hacia un objetivo (dirección) y persista en alcanzarlo (mantenimiento). Estos autores, luego de recopilar las opiniones de muchos otros, formulan la siguiente definición de motivación:

podríamos entenderla como proceso que explica el inicio, dirección, intensidad y perseverancia de la conducta encaminada hacia el logro de una meta, modulado por las percepciones que los sujetos tienen de sí mismos y por las tareas a las que se tienen que enfrentar (p. 5).

\section{Desarrollo histórico}

De acuerdo con las explicaciones brindadas por Herrera et al. (2004), la evolución histórica de la interpretación sobre la motivación puede resumirse de la siguiente manera:

- $\quad$ Entre la década de 1920 hasta mediados de la década de 1960, el tema de 
la motivación estuvo asociado con la investigación experimental, sobre aspectos como la conducta motora, el instinto y el impulso. En ese periodo, se buscaba determinar qué es lo que conduce a un organismo a restaurar $\mathrm{su}$ estado de equilibrio $\mathrm{u}$ homeostasis, con base en factores externos determinantes de la motivación, como los refuerzos.

- Después de la década de 1960, aparecieron las teorías cognitivas de la motivación, centradas en la experiencia consciente, el interés por la motivación de rendimiento y su importancia, junto con los logros en la vida personal. Por ejemplo, la teoría de Atkinson (citado por Herrera et al., 2004) señala que la motivación de rendimiento se determina por el valor otorgado a la meta y las expectativas por lograrla, teniendo en cuenta las características de las personas con alta o baja necesidad de rendimiento, ansiedad y control interno.

- A partir de la década de 1970 hasta la actualidad, la tendencia está marcada por las teorías cognitivas, en las que se destaca la importancia de algunos de sus elementos constitutivos; entre ellos, el autoconcepto, como elemento central de las teorías motivacionales. Diferentes estudios se refieren a aspectos tales como el papel de la atribución causal, la percepción de competencia, la percepción de control, las creencias sobre capacidad y autoeficacia y la indefensión aprendida, entre otros.

Las teorías sobre la motivación de base cognitiva tienen un gran valor para la educación porque facilitan el entendimiento de la conducta y el rendimiento escolar y permiten determinar estrategias para reforzar la motivación del estudiantado.

\section{Perspectivas teóricas sobre la motivación: La conductista, la humanista y la cognitiva}

DeacuerdoconSantrock(2002), existen tres perspectivas fundamentales respecto de la motivación: la conductista, la humanista y la cognitiva. La conductista subraya el papel de las recompensas en la motivación, la humanista en las capacidades del ser humano para desarrollarse y la cognitiva enfatiza en el poder del pensamiento.

\subsection{Perspectiva conductual}

Esta perspectiva señala que las recompensas externas y los castigos son centrales en la determinación de la motivación de las personas (Santrock, 2002). Las recompensas son eventos positivos o negativos que pueden motivar el comportamiento. Los que están de acuerdo con el empleo de incentivos recalcan que agregan interés y motivación a la conducta, dirigen la atención hacia comportamientos adecuados y la distancian de aquellos considerados inapropiados.

Trechera (2005) explica que las teorías que se basan en el empleo de incentivos parten del supuesto de que:

Las personas suelen realizar comportamientos con el objetivo de obtener algún beneficio y evitan o dejan de hacer aquellas conductas que conllevan un daño. Para este enfoque toda modificación de conducta se realiza básicamente a través de refuerzos, recompensas o mediante la evitación u omisión de aquello que sea desagradable (p. 3).

Para lograr la modificación de una conducta se pueden aplicar diferentes métodos, entre estos el reforzamiento, la extinción (no reforzar una conducta) y el castigo.

Los refuerzos pueden ser positivos o negativos. Los positivos se emplean para aumentar la probabilidad de que una respuesta esperada ocurra, por lo que 
puede decirse que son una recompensa. El principio del refuerzo positivo establece que: "si en una situación dada una persona hace algo que es seguido inmediatamente por un reforzador positivo, es más probable que esa persona haga de nuevo la misma cosa cuando se enfrente a una situación familiar" (Martín y Pear, citados por Naranjo, 2004, p. 41).

Naranjo (2004) señala que existen diversos factores que se deben tomar en cuenta para hacer un uso efectivo del reforzamiento positivo. En primer lugar, debe identificarse de forma específica el o los comportamientos que van a reforzarse. En segundo lugar, deben seleccionarse los motivadores apropiados. En tercer lugar, deben tomarse en cuenta otros aspectos como la inmediatez en la presentación del refuerzo, la privación y la saciedad, la cantidad de reforzamiento y la novedad del refuerzo.

El reforzador debe administrarse inmediatamente después de que se presenta la respuesta deseada. Cuando el reforzamiento se da de inmediato, la persona puede identificar más fácilmente las conductas por las que recibe el refuerzo y las que no. En cuanto a la privación y la saciedad, se considera que un reforzador se torna ineficaz si la persona no ha sido privada de él durante algún tiempo. Por ejemplo, ofrecer un aperitivo no funciona como reforzador si la persona acaba de comer. En este caso, hay un problema de saciedad; es decir, se ha experimentado tanto el reforzador que deja de cumplir su función. La novedad es otro aspecto importante a considerar en la motivación para lograr que los refuerzos sean más efectivos. Así, por ejemplo, podría utilizarse una sorpresa como refuerzo por emitir una conducta esperada.

Respecto del refuerzo negativo, se le conoce también como estímulo aversivo, y se emplea, al igual que el reforzamiento positivo, para aumentar o mantener una conducta. En el reforzamiento negativo se elimina un estímulo considerado aversivo (por ejemplo lavar los baños) para lograr una conducta. Para que los programas de reforzamiento negativo sean eficaces, siempre deben ir combinados con reforzamiento positivo.

En cuanto al método de extinción, consiste en dejar de reforzar una conducta. De acuerdo con Trechera (2005), se produce la extinción de una conducta cuando no se presenta el estímulo reforzador que la sustenta. Al no reforzar el comportamiento, este se irá debilitando, disminuyendo así la probabilidad de que se repita.

En el método de castigo se administra una consecuencia aversiva a la aparición de una conducta. Es una forma de que la persona aprenda lo que no tiene que hacer, en lugar de lo que tiene que hacer. Azaroff y Mayer (citados por Naranjo, 2004) señalan que:

El castigo es un procedimiento que por lo general deberá reservarse para las conductas inadaptadas serias, como la destructividad extrema hacia uno mismo u otras personas, y las conductas inadaptadas que interfieren persistentemente con el funcionamiento adaptado de clientes y compañeros (p. 52).

Se logra una desaparición más pronta de una conducta cuando se combina el castigo con la extinción. Valdés (2005) se refiere a algunas reglas para emplear las técnicas para la modificación de conducta, entre ellas:

- $\quad$ No recompensar por igual a todas las personas, los refuerzos o recompensas deben basarse en las cualidades y el desempeño personal; asegurarse de decir a la persona lo que debe hacer para obtener un reforzamiento.

- Indicar a la persona lo que está haciendo mal.

- No castigar en presencia de otras personas, pues la represión en público humilla; asimismo, ser justo al otorgar una recompensa.

García (2008) establece diferencias entre motivadores y motivaciones señalando, por una parte, que los motivadores son cosas que inducen a la persona a alcanzar un alto 
desempeño; por otra parte, las motivaciones son reflejo de los deseos del individuo, por lo que los motivadores son las recompensas o incentivos ya identificados que aumentan el impulso a satisfacer esos deseos. Un motivador es algo que influye poderosamente en la conducta de una persona.

Asimismo, se señalan diferencias entre motivación y satisfacción. La motivación se refiere al impulso y esfuerzo por lograr una meta, mientras que la satisfacción al gusto que se experimenta una vez alcanzada la meta. Es decir, la motivación implica un impulso hacia un resultado, en tanto que la satisfacción es la experiencia del resultado.

\subsection{Perspectiva humanista}

La perspectiva humanista enfatiza en la capacidad de la persona para lograr su crecimiento, sus características positivas y la libertad para elegir su destino. Dentro de esta perspectiva se ubica la Teoría de las necesidades. De acuerdo con García (2008), una de las teorías más conocidas sobre la motivación es la de la Jerarquía de las necesidades propuesta por Abraham H. Maslow, quien concibió las necesidades humanas ordenadas según una jerarquía donde unas son prioritarias y solo cuando estas están cubiertas, se puede ascender a necesidades de orden superior. Una vez satisfechas una serie de necesidades, estas dejan de fungir como motivadores. De acuerdo con la teoría de Maslow (citado por Santrock, 2002) las necesidades se satisfacen en el siguiente orden, primero las necesidades básicas y luego las necesidades más altas:

- Necesidadesfisiológicas: se relacionan con el ser humano como ser biológico. Son las necesidades básicas para el sustento de la vida. Las personas necesitan satisfacer unos mínimos vitales para poder funcionar, entre ellos: alimento, abrigo, descanso.
- $\quad$ Necesidades de seguridad: son aquellas que conducen a la persona a librarse de riesgos físicos, de lograr estabilidad, organizar y estructurar el entorno; es decir, de asegurar la sobrevivencia.

- $\quad$ Necesidades de amor y pertenencia (sociales): como seres sociales, las personas experimentan la necesidad de relacionarse con las demás, de ser aceptadas, de pertenecer. Se relacionan con el deseo de recibir el afecto de familiares, amigos y amigas y de una pareja.

- Necesidades de estima: se relacionan con el sentirse bien acerca de sí mismo, de sentirse un ser digno con prestigio. Todas las personas tienen necesidad de una buena valoración de sí mismas, de respeto, de una autoestima positiva, que implica también la estima de otros seres humanos.

- Necesidades de autorrealización: de acuerdo con Valdés (2005) se les conocen también como necesidades de crecimiento, de realización del propio potencial, de realizar lo que a la persona le agrada y poder lograrlo. Se relacionan con la autoestima. Entre estas pueden citarse la autonomía, la independencia y el autocontrol.

Trechera (2005) señala:

El proceso de maduración humana se enriquece durante toda la vida. Siempre podemos desarrollar nuevas posibilidades. Esta necesidad se caracterizaría por mantener viva la tendencia para hacer realidad ese deseo de llegar a ser cada vez más persona (p. 2).

La insatisfacción de las necesidades señaladas en esta jerarquía tiene un impacto emocional importante, según indica Bisquerra (2000). Por otra parte, Valdés (2005) explica que Maslow descubrió dos necesidades adicionales que experimentan aquellas personas que han satisfecho las cinco mencionadas anteriormente (muy pocas personas según 
el autor), a las que llamó cognoscitivas, estas son:

- $\quad$ Necesidades de conocer y entender el mundo que rodea a la persona y la naturaleza.

- Necesidad de satisfacción estética, referida a las necesidades de belleza, simetría y arte en general.

\subsubsection{Teoría: Existencia, Relación y Crecimiento (E.R.G.)}

Esta teoría fue desarrollada por Clayton Alderfer (citado por Trechera, 2005). No se basa en elementos nuevos, sino que se fundamenta en la Teoría de la jerarquía de las necesidades de Maslow. El autor agrupa las necesidades en tres tipos:

- Existencia: son aquellas necesidades básicas y materiales que se satisfacen mediante factores externos y corresponden a las necesidades fisiológicas y de seguridad.

- Relación: requieren para su satisfacción de las relaciones interpersonales y de la pertenencia a un grupo. Se refieren a las necesidades sociales y de aceptación.

- Crecimiento: son las necesidades de desarrollo personal. Se satisfacen cuando la persona logra objetivos importantes para su proyecto vital, e incluyen el reconocimiento, la autoestima y la autorrealización.

Trechera (2005) menciona que, a pesar de que Alderfer basó su teoría en la de Maslow, la cuestiona porque considera que existe un movimiento ascendente en el modelo piramidal que denomina satisfacción progresiva y otro que conduce a la persona hacia atrás, al que llama frustración regresiva. Es decir, si alguien se frustra por no poder satisfacer ciertas necesidades, retrocederá para satisfacer necesidades inferiores.

\subsubsection{Teoría de las necesidades de McClelland}

McClelland (citado por Hampton, Summer y Webber, 1989) señala que existen tres motivaciones particularmente importantes: la necesidad de logro, la de afiliación y la de poder. Estas motivaciones son importantes porque predisponen a las personas a comportarse en formas que afectan de manera crítica el desempeño en muchos trabajos y tareas. Por su parte, García (2008) aduce que las anteriores son motivaciones sociales que se aprenden de una manera no consciente, como producto de enfrentarse activamente al medio. Explica este autor que las recompensas que suceden a una conducta la refuerzan, lo que aumenta la probabilidad de que se repita. Cuando el comportamiento de las personas opera en un ambiente propicio para obtener resultados satisfactorios, se aprende algo más que la respuesta a un problema, puesto que la forma de comportamiento asociado con el éxito también se ve reforzada.

Parece que en condiciones que estimulan la independencia y moderan la toma de riesgos, la persona adquiere interés por retos de proporciones manejables, que tal vez la conduzcan a experimentar altos sentimientos de logro. De igual forma, una necesidad fuerte de afiliación o de poder podría ser producto de una historia de recompensas asociadas con el comportamiento sociable o dominante.

Según lo mencionado, las necesidades de logro, de afiliación y de poder son fuertes o débiles dependiendo de asociaciones pasadas con el desempeño y las recompensas obtenidas en la solución de situaciones. Lawrence y Lorsch (citado por García, 2008) explican este proceso indicando que conforme la persona lucha por dominar los problemas, surgen ciertos comportamientos que resultan muy compensatorios, por cuanto proporcionan soluciones a estos. Como resultado, la próxima vez que la persona necesite enfrentar una situación, 
intentará emplear de nuevo el mismo esquema de comportamiento, pues ha aprendido a confiar en él. De esta forma, se dice que una persona está altamente motivada a competir (necesidad de logro), o que tiene mucha necesidad de relaciones sociales (necesidad de afiliación), como resultado de ese proceso de aprendizaje.

Existe otro elemento de gran importancia en el proceso de aprendizaje y motivación: la ansiedad. Se considera que se aprende no solo cuando el comportamiento va seguido de estados positivos o recompensas, sino también cuando los sucede una reducción de la tensión. García (2008) explica:

La reducción de ansiedad asociada con el comportamiento que ayuda a resolver los problemas contribuye en parte a reforzar dicho comportamiento. Si competir contra un estándar de excelencia recompensa al individuo reduciendo la ansiedad, la necesidad de logro se fortalece. Si las relaciones afectuosas y amistosas con otros van asociadas al éxito, la necesidad de afiliación se fortalece; y si la persuasión y el dominio están asociadas con el éxito, se fortalece la necesidad de poder (p. 6).

A continuación se explican con más detalle las tres motivaciones principales a las que se refiere la teoría de McClelland: las necesidades de logro, de afiliación y de poder.

\section{Necesidad de logro}

Autores como García (2008), Hampton et al. (1989), Trechera (2005) y Valdés (2005) mencionan que las personas con una alta necesidad de logro presentan características como las siguientes:

- Les gustan las situaciones en las que pueden tomar personalmente la responsabilidad de encontrar la solución a los problemas.

- Tienden a fijarse metas moderadas y a tomar riesgos calculados.

- Desean una retroalimentación concreta acerca de qué tan adecuadamente se están desempeñando.
- $\quad$ Se distinguen por intentar hacer bien las cosas, tener éxito, incluso por encima de las recompensas.

Dwek, Henderson y Leggett (citados por Santrock, 2002) identifican tres tipos de orientación al logro: la pericia, la incapacidad y la ejecución. Estos investigadores han encontrado, en el caso concreto de la población estudiantil, que los educandos muestran dos respuestas distintas ante situaciones que consideran desafiantes o difíciles, una de pericia o una de incapacidad. Las personas con una orientación de pericia se centran en la tarea y no en su habilidad, disfrutan del desafío y elaboran estrategias dirigidas a la solución, lo cual mejora su ejecución. Por el contrario, las que tienen una orientación de incapacidad se centran en sus insuficiencias personales, generalmente atribuyen sus dificultades a una falta de habilidad y son personas que pueden sentirse aburridas o ansiosas, lo cual deteriora su ejecución.

La orientación de ejecución de una tarea significa preocuparse por el resultado y no por el proceso. Para las personas orientadas hacia la ejecución, ganar es lo que importa y consideran que la felicidad es el resultado del hecho de ganar. No obstante, si no confían en su éxito enfrentan el problema de que si intentan y fracasan, con frecuencia asumen el fracaso como evidencia de su poca habilidad. Si no realizan ningún intento, tal vez encuentren una explicación más aceptable sobre su fracaso. A veces esta situación lleva a la persona a manifestar comportamientos que la protejan de tener una imagen de incompetencia a corto plazo, pero que interfiere con su aprendizaje y logro a largo plazo. De esta forma algunas personas para no tener que atribuir el fracaso a una falta de habilidad simplemente no intentan o copian, dan excusas, trabajan con poco entusiasmo o se fijan metas poco realistas. 


\section{Necesidad de poder}

Se considera que las personas que tienen una alta necesidad de poder, emplean más tiempo y esfuerzo pensando cómo obtener y ejercer el poder y la autoridad, que aquellas que tienen una baja necesidad de poder. Estas personas consideran que siempre necesitan ganar argumentos, persuadir a otras, hacer que sus criterios prevalezcan y se sienten incómodas sí no poseen ciertas cuotas de autoridad.

McClelland (citado por García, 2008) expresa que el poder realmente tiene dos caras. La primera origina reacciones negativas. Esta cara del poder se relaciona con situaciones de dominio-sumisión, con personas que piensan que debe hacerse lo que ellas desean y que pueden controlar a las demás. La otra cara del poder es positiva. Refleja el proceso mediante el cual el comportamiento persuasivo e inspirador de una persona puede evocar sentimientos de fortaleza y habilidad en otras y las ayuda a establecer metas. Es decir, cumple con el papel de apoyar a otras personas para que puedan expresar sus propias capacidades y de este modo, lograr metas que se propongan.

\section{Necesidad de afiliación}

Siguiendo las explicaciones de McClelland (citado por Hampton et al., 1989 y en García, 2008), las personas con una alta necesidad de afiliación tienden a interesarse y a pensar con frecuencia acerca de la calidad de sus relaciones personales. Conceden mucha importancia a los momentos agradables que comparten con otras personas y se preocupan por las deficiencias o dificultades en sus relaciones con otras. La meta común de la motivación de afiliación es la interacción social y lograr la aceptación de otras personas. En ciertos casos, el comportamiento de afiliación se relaciona con una reducción de la ansiedad; en otros, contribuye más a que la persona se asegure la aprobación social de sus puntos de vista.

Independientemente de los orígenes de la necesidad de afiliación, esta tiende a producir esquemas de comportamiento similares. Las personas con una gran necesidad de afiliación buscan la compañía de otras, toman medidas e ingenian estrategias para ser admitidas por estas, intentan proyectar una imagen favorable en sus relaciones interpersonales, moderan las tensiones desagradables en sus entrevistas, ayudan y apoyan a otras, y desean ser admiradas en retribución.

En relación con las personas adolescentes, específicamente los y las estudiantes, los cambios evolutivos en las relaciones con los iguales tienden a influir en la motivación hacia la institución educativa y su implicación en ella, según señala Rice (2000). Existe un aumento en la importancia que se atribuye a amigas y amigos durante la transición a la adolescencia y durante esta. La inclusión, aceptación y aprobación del grupo de iguales tiene una marcada influencia sobre la motivación de logro. Goodenow (citado por Rice, 2000) menciona que esa pertenencia está definida como el sentimiento que tiene la persona de ser valorada, incluida y motivada por otras; por ejemplo, profesoras y profesores, compañeras y compañeros, en el contexto de la clase y sentirse una parte importante de la vida y actividad en las lecciones.

\section{Necesidad de autosuficiencia}

Bandura (1997) se refiere al concepto de autosuficiencia como la creencia que tiene la persona de que puede dominar una situación y lograr resultados positivos. Considera que la autosuficiencia es un factor determinante en el éxito académico del estudiantado. Se concreta en creencias tales como que se es una persona capaz que puede rendir en diferentes actividades. Por otra parte, es probable que las personas 
con baja autosuficiencia en el aprendizaje eviten ciertas tareas, sobre todo aquellas que son desafiantes, mientras que aquellas con alta autosuficiencia trabajan con entusiasmo en actividades de aprendizaje.

Sentirse autosuficiente en una variedad de situaciones ofrece como recompensa una mejoría en la autoestima y la motivación. El sentido de competencia es fuerte o débil dependiendo del equilibrio de éxitos y fracasos que se haya experimentado. Abarca (2001) menciona que la motivación no es algo estático, sino que está en constante transformación, evoluciona y que existe una relación entre su desarrollo y las experiencias que cada persona enfrenta en el diario vivir.

\subsection{Perspectivas cognitivas}

Las teorías cognitivas enfatizan que lo que la persona piensa sobre lo que puede ocurrir es importante para determinar lo que efectivamente sucede (Ajello, 2003). El sistema cognitivo es el que recibe y envía información a los otros sistemas: afectivo, comportamental y fisiológico, y regula el comportamiento de estos poniendo en marcha o inhibiendo ciertas respuestas en función del significado que le da a la información de que dispone. De esta forma, las ideas, creencias y opiniones que tenga la persona sobre sí y sobre sus habilidades determinan el tipo y la duración del esfuerzo que realiza y, por tanto, el resultado de sus acciones.

Esas tesis son compartidas por Santrock (2002), quien indica que, de acuerdo con la perspectiva cognitiva, los pensamientos, en el caso concreto de la persona estudiante, guían su motivación. De acuerdo con Pintrich, Schunk, Ertmer y Zimmerman (citados por Santrock, 2002), el interés que ha surgido respecto de la teoría cognitiva se centra en ideas tales como la motivación interna de logro de las personas, sus atribuciones acerca del éxito o del fracaso y sus creencias sobre lo que pueden controlar de forma efectiva en su ambiente, de igual manera que la importancia del establecimiento de metas, el planeamiento y el monitoreo del progreso hacia una meta.

\subsubsection{Teoría de las expectativas de Vroom}

García (2008), Trechera (2005) y Valdés (2005) señalan que esta teoría o modelo de la motivación fue elaborada por Víctor Vroom, quien considera que las personas se motivan a realizar cosas y esforzarse por lograr un alto desempeño para alcanzar una meta si creen en su valor, si están seguras de que lo que harán contribuirá a lograrla y si saben que una vez que alcancen la meta recibirán una recompensa, de tal manera que el esfuerzo realizado ha valido la pena. García (2008) explica:

En mayor detalle, la teoría de Vroom señala que la motivación de las personas para hacer algo estará determinada por el valor que asignen al resultado de su esfuerzo (ya sea positivo o negativo), multiplicado por la confianza que tienen de que sus esfuerzos contribuirán materialmente a la consecución de la meta (p. 11).

Para Vroom (1964), la motivación es el resultado de tres variables: valencia, expectativas einstrumentalidad. Lavalencia se refiere al valor que la persona aporta a cierta actividad, el deseo o interés que tiene en realizarla. Las expectativas se definen como las creencias sobre la probabilidad de que un acto irá seguido de un determinado resultado. La instrumentalidad se refiere a la consideración que la persona hace respecto de que si logra un determinado resultado, este servirá de algo. De acuerdo con lo anterior, si una persona no se siente capaz, piensa que el esfuerzo realizado no va a tener repercusión o no tiene interés por la tarea, no tendrá motivación para llevarla a cabo.

Porter y Lawer elaboraron un modelo de motivación más completo basado en la 
Teoría de las expectativas de Vroom (García, 2008) que propone que el desempeño en la realización de tareas o el cumplimiento de metas está determinado esencialmente por el esfuerzo aplicado. También, influye la habilidad de la persona, sus conocimientos y destrezas para realizar el trabajo y su percepción de lo que es la tarea requerida. El desempeño permite obtener recompensas intrínsecas, tales como la sensación de logro o autorrealización, y recompensas extrínsecas, como el prestigio.

\subsubsection{Teoría de la equidad de Stacey Adams}

Valdés (2005) indica que esta teoría de la motivación se enfoca en el criterio que se forma la persona en función de la recompensa que obtiene comparándola con las recompensas que reciben otras personas que realizan la misma tarea o hacen aportes semejantes. Trechera (2005) agrega que para establecer ese criterio la persona toma en cuenta dos tipos de elementos:

- Inputs: las contribuciones que la persona aporta a la tarea; a saber, formación, capacidad, experiencia, habilidad, nivel de esfuerzo, tareas realizadas, entre otras.

- Outputs: los resultados, las ventajas o beneficios que la persona obtiene por la actividad realizada.

Esta teoría es importante por cuanto considera fundamental la percepción que el individuo tenga respecto de la experiencia que ejecuta o pretende ejecutar.

\subsubsection{Modelo de fijación de metas u objetivos}

Una meta $u$ objetivo es aquello que la persona intenta alcanzar; es decir, el fin de una acción (Trechera, 2005). Los factores que debe contemplar un objetivo para que motive son:

- $\quad$ El conocimiento, ya que se debe conocer la meta y los medios requeridos para alcanzarla.

- La aceptación, debido a que debe existir acuerdo sobre lo que se desea realizar.

- La dificultad, que significa que las metas deben ser difíciles, pero no imposibles. Deben suponer un reto, pero no han de ser inalcanzables.

- La especificidad, puesto que cuanto más concreto sea el objetivo, más fácil será aportar el esfuerzo para lograrlo.

Lockey Latham (citados por Trechera, 2005) indican que en el establecimiento de metas se debe resaltar que el objetivo es el que orienta la acción; es decir, anima a realizar estrategias y planes de acción. Al centrar la atención, la persona selecciona qué actuaciones son importantes y se conduce en consecuencia. Para lograr una meta se tienen que plantear las alternativas idóneas. El objetivo, a su vez, sirve para regular el esfuerzo. Al tener claro qué es lo que se debe hacer, la persona puede programar sus acciones más fácilmente para lograrlo. Asimismo, el objetivo permite que se trabaje de una manera más persistente; además, se presenta como aquello que con esfuerzo y persistencia se puede alcanzar.

Valdés (2005) señala que las teorías sobre motivación pueden clasificarse en dos grandes grupos: las de contenido y las de proceso. Las primeras estudian y consideran aspectos que pueden motivar a las personas, tales como las necesidades, las aspiraciones y el nivel de satisfacción. Entre estas teorías se pueden mencionar las de la Pirámide de Necesidades de Abraham Maslow, las teorías ERG (Existence, Relatedness and Growth) de Clayton Alderfer y la Teoría de las necesidades de McClelland. Las segundas estudian y tienen en cuenta el proceso de pensamiento por el cual la 
persona se motiva. Entre estas teorías destacan la de las Expectativas de Víctor Vroom, la Teoría de la Equidad de Stacey Adams y la Teoría de la Modificación de la Conducta de Skinner.

Trechera (2005) se refiere también a las teorías anteriores llamándolas modelos explicativos, los cuales clasifica en tres grandes categorías: modelos basados en la satisfacción de necesidades (Maslow, Alderfer y McClelland), modelo basado en los incentivos (Skinner) y modelos cognitivos (Vroom y Adams).

\section{4. Ámbito Educativo}

\subsection{Relación entre Emoción y Motivación}

Una razón importante para analizar el tema de la motivación es su incidencia en el aprendizaje. Una de las propuestas que mejor plantea la complejidad de los procesos motivacionales académicos, según Cerezo y Casanova (2004), es la que presentan Pintrich y De Groot, que distinguen tres categorías relevantes para la motivación en ambientes educativos: la primera se relaciona con un componente de expectativas, que incluye las creencias de las personas estudiantes sobre su capacidad para ejecutar una tarea; la segunda se asocia a un componente de valor, relacionado con sus metas y sus percepciones sobre la importancia e interés de la tarea; la tercera, a un componente afectivo, que incluye las consecuencias afectivo-emocionales derivadas de la realización de una tarea, así como de los resultados de éxito o fracaso académico. Estos autores agregan que las investigaciones revelan que la persona se motiva más por el proceso de aprendizaje cuando confía en sus capacidades y posee altas expectativas de auto-eficacia, además de valorar las actividades educativas y responsabilizarse de los objetivos de aprendizaje.
Sobre el tema de la emoción, diversos autores mencionan que existe un vínculo entre esta y la motivación. Así por ejemplo, explica Bisquerra (2000) que:

Desde la neurofisiología también se ha comprobado la relación entre emoción y motivación. Las estructuras neuronales y los sistemas funcionales responsables de la motivación y de la emoción, a menudo coinciden, conformando un cerebro motivacional/emocional conocido con el nombre de Sistema Límbico (p. 166).

Cuando se genera una emoción se produce una predisposición a actuar. Es una motivación reactiva; es decir, la conducta es motivada como respuesta a las condiciones del medio. La emoción depende de lo que es importante para la persona.

Alonso (1992) se refiere a dos problemas motivacional-afectivos que enfrentan con frecuencia algunas personas estudiantes, los cuales pueden atribuirse a las condiciones poco favorables en la institución educativa y al uso incorrecto de la dimensión afectiva por parte del personal docente. Estos problemas motivacional-afectivos se refieren a la denominada indefensión y a la desesperanza aprendida. La indefensión aparece como un patrón de comportamiento entre los once y los doce años de edad. Estudiantes con este comportamiento atribuyen el éxito académico a causas externas, cambiantes y fuera de su control. Asimismo, señalan autocogniciones negativas y manifiestan que su inteligencia, memoria o su capacidad para resolver problemas son deficientes. Experimentan aburrimiento o ansiedad frente a las actividades educativas y a medida que aumentan las situaciones en que fracasan, disminuye su participación académica.

La desesperanza aprendida se puede observar en niñas y niños a partir de los ocho o nueve años de edad. No importa si obtienen logros académicos, estas personas piensan y sienten que están destinadas a fracasar. Quienes manifiestan indefensión y desesperanza aprendida tienden a ver sus propias dificultades como fracasos insuperables, debido a sus limitadas habilidades. 


\subsection{Teoría de las atribuciones y desempeño académico}

Las atribuciones se consideran como las causas percibidas de un comportamiento. Graham y Weimer (citados por Santrock, 2002) mencionan que la búsqueda de una causa o explicación empieza generalmente cuando diferentes eventos inesperados o importantes terminan en fracaso; por ejemplo, cuando se obtiene una calificación baja. Sobre este tema, Heider, autor de la Teoría de la atribución (citado por Bisquerra, 2000), propuso que las personas realizan atribuciones de causalidad que tienen efectos sobre las emociones, el pensamiento, la motivación, el comportamiento y la autoestima. En esta teoría las emociones se consideran el resultado de atribuciones causales que inciden sobre las expectativas de éxito y, por ende, en la motivación que activa el comportamiento. De este modo, las expectativas altas facilitan la motivación y las bajas la desmotivación.

Bisquerra (2000) menciona que pueden distinguirse cuatro tipos de estilo atributivo:

a. externalista: tanto los éxitos como los fracasos se atribuyen a causas externas (ambiente, otros);

b. internalista: tanto los éxitos como los fracasos se atribuyen a causas internas de la persona (capacidad, esfuerzo);

c. egoísta: los éxitos se atribuyen a causas internas y los fracasos a causas externas ("he aprobado", "me han suspendido"); y,

d. depresivo: los éxitos se atribuyen a causas externas y los fracasos a factores internos.

Heider (citado por Bisquerra, 2000) presenta también diversas dimensiones de la atribución causal: situación, tiempo y control; estas se manifiestan así:

\section{Situación:}

a. Global: "No he aprobado el examen porque en todos salgo mal".

b. Específica: "He hecho mal este examen y por eso he reprobado".

\section{Tiempo:}

a. Estable: "Hice mal el examen porque siempre lo hago mal".

b. Inestable: "No me he sentido bien y por eso salí mal en el examen".

\section{Control:}

a. Incontrolable: "Salí mal en el examen porque no soy capaz".

b. Controlable: "Salí mal en el examen porque el ruido no me dejó concentrarme".

Las atribuciones inapropiadas se sitúan en las dimensiones global, estable o incontrolable y pueden darse en una de estas dimensiones, en una combinación de ellas e, incluso, en las tres al mismo tiempo. Estas atribuciones tienen un impacto emocional negativo y dan como resultado un fracaso en la motivación, por lo que es deseable que las atribuciones sean específicas, inestables y controlables.

Graham y Weimer (citados por Santrock, 2002) señalan que la percepción que la persona estudiante tiene sobre la estabilidad de una causa incide en sus expectativas de éxito. Si atribuye un resultado positivo a una causa estable, como su capacidad, esperará éxitos futuros. Por el contrario, si atribuye un resultado negativo a una causa estable, esperará fracasos en el futuro. Si la atribución de los fracasos se refiere a causas inestables como la mala suerte o la falta de esfuerzo, la persona podría tener expectativas de lograr ser exitosa en el futuro, debido a que percibe que la causa de su fracaso es modificable.

Los autores mencionados explican que la percepción acerca del control de la 
causa está asociada con diversos estados emocionales como la ira, la culpa, la compasión y la vergüenza. Cuando las personas estudiantes perciben que las causas que no les permiten tener éxito se deben a factores externos que otras podrían controlar, con frecuencia se sienten enojadas. Cuando perciben que no se han desempeñado de forma adecuada debido a causas internas controlables como la falta de esfuerzo, pueden sentirse culpables. Cuando piensan que otras personas no logran sus metas debido a causas incontrolables como por ejemplo la falta de habilidad, sienten compasión o simpatía. Cuando fracasan debido a factores internos incontrolables, experimentan pena, humillación y vergüenza.

Santrock (2002), relaciona estos aspectos de la motivación con la autoestima y señala que:

La percepción que tiene el alumno del éxito o fracaso en función de factores internos y externos, influye en su autoestima. Los estudiantes que perciben que su éxito se debió a razones internas tales como el esfuerzo, tienen más posibilidades de tener una autoestima más alta después del éxito, que aquellos alumnos que creen que su éxito se debió a razones externas, como la suerte. Cuando se trata de analizar el fracaso, las atribuciones internas llevan a una disminución en la autoestima (p. 440)

Debido a su compleja naturaleza, la motivación tiene una amplia relación con otros conceptos vinculados con la dirección y la intensidad de la conducta, como son el interés, la necesidad, el valor, la actitud y la aspiración. El interés se refiere a una atención selectiva. La necesidad implica carencia de algo que puede ser obtenido mediante una actividad. El concepto de valor implica una orientación a la meta o metas principales en la vida de la persona. En la actitud se distinguen tres componentes: el cognitivo, ideas o creencias acerca de algo o alguien; el emocional, sentimientos de agrado o desagrado, y el conductual, que es una conducta relacionada con el objeto de la actitud. Finalmente, la aspiración se refiere a la expectativa de alcanzar un determinado nivel de logro. El interés, la necesidad, el valor, la actitud y la aspiración dirigen la conducta y controlan la intensidad de esta en la dirección señalada (Herrera et al., 2004).

\subsection{Motivos de la población estudiantil}

Algunos de los motivos que orientan el comportamiento se describen (de la Herrán Gastón, 2008), como:

- Relacionados con la seguridad emocional: necesidad de estar emocionalmente segura o seguro, libre de riesgos, amenazas, castigos, humillaciones.

- Relacionados con las sensaciones: necesidad de placer, de experimentar emociones, de relajación.

- Relacionados con el esfuerzo: necesidad de reconocimientos, trato cordial, contacto físico, tiempo y atención.

- Relacionados con personas y grupos significativos: necesidad de respeto, aceptación e integración, de entablar amistad, de querer y ser querida o querido.

- Relacionados con la autoestima: necesidad de ser apreciada o apreciado, de experimentar éxito, de sentirse valiosa o valioso.

- Cognoscitivos: necesidad de entender, saber, descubrir, crear, explorar, afrontar retos, obtener satisfacción por medio del aprendizaje, tomar decisiones autónomas, lograr la realización de planes y la resolución de problemas.

- Estéticos: búsqueda del orden, de la estructura, de la belleza.

- Auto-constructivos y de mejora social: necesidad de intimidad, compromiso, independencia, autodeterminación, búsqueda de madurez y de desarrollo de las propias capacidades. 
De acuerdo con lo analizado sobre diferentes aspectos que influyen en la motivación del estudiantado, podrían sugerirse algunas acciones para realizar en el ámbito educativo, como las siguientes:

- Ayudar a la persona estudiante a descubrir y confiar en sus capacidades y habilidades eliminando ideas erróneas que pueda tener respecto de su auto-eficacia.

- Analizar la importancia de las actividades educativas y del aprendizaje en general para la vida.

- $\quad$ Facilitar experiencias de logro académico que favorezcan el autoconcepto y la autoestima.

- Promover ambientes educativos que beneficien la dimensión afectiva, lo que evita así la aparición del aburrimiento, el estrés o la ansiedad que aumentan los sentimientos e ideas de fracaso y menoscaban la valoración personal y el rendimiento académico.

- Permitir la expresión de emociones que se viven cuando se enfrentan situaciones de fracaso y ayudar a eliminar aquellas ideas negativas o irracionales que podrían estar sustentándolas, de modo que la autoestima no se lesione.

- Eliminar las amenazas, castigos y humillaciones y, por el contrario, brindar espacios para la atención personal que permitan experimentar aceptación y respeto.

- Propiciar situaciones placenteras de tranquilidad y éxito, de modo que la persona se sienta segura y valiosa.

- Desarrollar procesos de enseñanzaaprendizaje que despierten el deseo de descubrir, conocer, experimentar y crear.

- Promover experiencias propicias para el desarrollo de la independencia, la resolución adecuada de problemas y la toma de decisiones autónomas.

\subsection{Motivaciones intrínseca $y$ extrínseca en estudiantes}

Ajello (2003) señala que la motivación intrínseca se refiere a aquellas situaciones donde la persona realiza actividades por el gusto de hacerlas, independientemente de si obtiene un reconocimiento o no. La motivación extrínseca, por su parte, obedece a situaciones donde la persona se implica en actividades principalmente con fines instrumentales o por motivos externos a la actividad misma, como podría ser obtener una recompensa.

La perspectiva conductual enfatiza la importancia de la motivación extrínseca (Santrock, 2002). Esta motivación incluye incentivos externos, tales como las recompensas y los castigos. De forma diferente, las perspectivas humanista y cognitiva enfatizan la importancia de la motivación intrínseca en el logro. La motivación intrínseca se fundamenta en factores internos, como la autodeterminación, la curiosidad, el desafío y el esfuerzo. Respecto de estos tipos de motivación, se puede observar que ciertas personas se aplican en sus estudios, porque desean obtener buenas calificaciones o para evitar la desaprobación de la madre o el padre; es decir, están motivadas extrínsecamente. Otras lo hacen porque están motivadas internamente a obtener niveles altos de desempeño académico.

La motivación interna y el interés intrínseco en las actividades académicas aumentan cuando la persona tiene posibilidades de elección y oportunidades para tomar la responsabilidad personal de su aprendizaje, establecer sus propias metas, planear cómo alcanzarlas y monitorear su progreso. Por otra parte, cuando sus habilidades son altas, pero las actividades no son desafiantes, el resultado es el aburrimiento. Cuando el desafío y los niveles de habilidad son bajos, se experimenta apatía y cuando se enfrenta una tarea desafiante para la que no se cree tener las habilidades necesarias, se experimenta ansiedad. 
Eccles (citado por Universidad de Sevilla, 2005) sugiere que la falta de ajuste entre el ambiente de la institución secundaria y las necesidades de las personas adolescentes, contribuye a un cambio negativo observado en la motivación. El personal docente se vuelve más controlador cuando las personas requieren más autonomía y las relaciones interpersonales se transforman en más distantes cuando se necesita de más apoyo.

Estudios realizados por Harter (citado por Universidad de Sevilla, 2005) revelan que la orientación motivacional está altamente relacionada con la percepción de competencia educativa. De esta forma, las personas que evalúan su competencia académica de manera positiva generalmente reportan estar motivadas intrínsecamente, mientras que las percepciones negativas se asocian con una motivación extrínseca. Los estudios realizados por este autor también han mostrado que la motivación intrínseca decae a lo largo de los años de colegio.

Para determinar por qué se pierde la motivación intrínseca, Harter (citado por Universidad de Sevilla, 2005) aplicó a estudiantes un cuestionario basado en dos factores: evaluación externa y comparación social. Las dimensiones incluidas en la evaluación externa fueron el énfasis en las notas por parte del personal docente, la competición, el control de elección y el interés social. Este autor encontró que al aumentar el nivel, el estudiantado percibe que el profesorado pone un mayor énfasis en las notas y se centra más en la competición y el control, lo cual realza de esta manera la evaluación externa del desempeño y, al mismo tiempo, presta poca atención a los intereses personales de la población estudiantil.

Los resultados también muestran que las personas piensan que a mayor énfasis en los componentes de la evaluación externa, más incapaces se sienten y eso va en detrimento de su motivación intrínseca. El aumento del énfasis en la comparación social con sus iguales fomenta también la reevaluación de la competencia académica, lo que puede tener efectos psicológicos perjudiciales para un gran número de estudiantes porque podrían concluir que son incompetentes.

De acuerdo con Moss (citado por Ajello, 2003), la satisfacción de las personas estudiantes, su crecimiento personal y su rendimiento académico son óptimos solo si el afecto del profesor o profesora y su apoyo van acompañados de una eficiente organización, de la constante atención de los aspectos de tipo académico y del planeamiento de clases bien estructuradas y con objetivos claros.

\section{Conclusiones}

Existen tres perspectivas fundamentales sobre la motivación: la conductista, la humanista y la cognitiva. La perspectiva conductual enfatiza que las recompensas motivan la conducta y dirigen la atención de las personas hacia acciones adecuadas y la distancian de las inadecuadas. La perspectiva humanista subraya la capacidad humana para crecer, las cualidades personales y la libertad de elección. La teoría cognitiva enfatiza en las ideas y considera que lo que la persona piensa que puede ocurrir es importante porque determina lo que ocurre.

Cada una de las perspectivas teóricas brinda valiosos aportes que permiten comprender de manera más integral cómo opera el fenómeno de la motivación en el ser humano. Se puede entender, por consiguiente, que la motivación está influida por múltiples variables; por ejemplo, el impacto de los refuerzos positivos y negativos sobre el comportamiento, al que se refiere el enfoque conductual; todas las necesidades $\mathrm{y}$ emociones que considera el enfoque humanista y las ideas, creencias, opiniones y expectativas que toma en cuenta el enfoque cognitivo. Estas posiciones teóricas se complementan, pues consideran diferentes dimensiones del funcionamiento humano. Las personas son seres con conductas, emociones, necesidades, pensamientos, y todos estos se relacionan entre sí. Lazarus (citado por Naranjo, 2004) opina que la singularidad del pensamiento es un gran impedimento 
para el aprendizaje y el progreso. Existe un deseo de hallar una solución verdadera, precisa y perfecta a los problemas humanos; sin embargo, no existen causas únicas para estos.

Un objetivo fundamental que debe proponerse en el ámbito educativo es lograr la motivación del estudiantado en relación con el aprendizaje, por cuanto, como se mencionó, la motivación activa dirige y mantiene la conducta hacia las metas educativas que este se propone. El grado de motivación que se obtenga debe ser lo suficientemente relevante para que la persona desarrolle la disposición para aprender y continúe haciéndolo por sí misma, por su propio gusto y para su crecimiento académico y personal.

Existen tres aspectos relevantes para la motivación que deben tomarse en cuenta en el ámbito educativo: las expectativas de la población estudiantil, el valor otorgado a las metas educativas y las consecuencias afectivo-emocionales resultantes del éxito o del fracaso académico. De igual forma, deben tenerse presentes los problemas motivacional-afectivos: la indefensión y la desesperanza aprendida, los cuales son patrones de comportamiento que surgen desde la infancia, en los que las personas perciben sus fracasos como insuperables porque consideran que carecen de habilidades.

El estudiantado realiza atribuciones de causalidad ante situaciones que le ocurren, las cuales repercuten en sus emociones, pensamiento, motivación, comportamiento y autoestima. Es importante que la estudiante y el estudiante aprendan a modificar su percepción o las razones que suelen atribuir como causas de sus éxitos o fracasos académicos, de manera que puedan identificar causas permanentes y generales para aquellos eventos positivos (por ejemplo; me desempeñé bien en la prueba porque soy una persona capaz) y, por el contrario, sean capaces de encontrar causas específicas y temporales para eventos negativos (por ejemplo; no salí bien en el examen porque no estudié lo suficiente).
Considerar algunos aspectos como los siguientes puede ayudar a las personas estudiantes a no desmotivarse:

- $\quad$ Comprender que es natural no poseer la misma capacidad para desempeñarse en las diversas áreas del aprendizaje; por ello es importante aprender a conocerse y realizar una valoración objetiva tanto de las fortalezas propias para aprovecharlas, así como de las debilidades para identificar dónde se requiere mayor esfuerzo y del progreso que se va obteniendo.

- $\quad$ Entender que equivocarse y aprender de los errores forma parte del proceso de aprendizaje formal y de la vida en general. Igualmente, comprender que en muchas ocasiones es importante y conveniente solicitar ayuda a fin de evacuar dudas o recibir más información que facilite comprender y asimilar de mejor manera el conocimiento, luego de entender que pedir ayuda es una necesidad y un derecho de todas las personas.

- Comprender que la inteligencia no es un rasgo fijo, sino que más bien se puede cultivar mediante el aprendizaje y que por lo tanto hay posibilidades de cambio y de mejoría en el desempeño académico.

Para toda institución educativa resulta de gran importancia conocer los posibles motivos que guían la conducta de la población estudiantil y que están relacionados con la seguridad emocional, las sensaciones, el refuerzo, las personas y los grupos significativos, la autoestima, y los aspectos cognoscitivos y estéticos, autoconstructivos y de mejora social. Este conocimiento permite el desarrollo de acciones que favorezcan la motivación hacia el aprendizaje.

Debe recordarse que, cuando se expone a estudiantes a desafíos académicos que están fuera de su alcance, es probable que se frustren y se llenen de 
tensión; por otra parte, si los desafíos son pobres les provoca aburrimiento. Ambas situaciones producen desmotivación, por lo que resulta recomendable que las exigencias académicas estén acordes con las capacidades del estudiantado y se adecuen a sus etapas de desarrollo.

Es importante que el personal docente trate de despertar la curiosidad del estudiantado con respecto a los diferentes temas que se analizan con el propósito de captar su interés y atención sobre estos. Algunos aspectos que podrían resultar valiosos para lograr tal fin son: la novedad de los conocimientos y el destacar su importancia, de modo que la persona estudiante pueda encontrarle sentido al aprendizaje; asimismo, promover la exploración y reflexión de temas de su interés y la integración de los nuevos conocimientos con aquellos previos.

Se debe estimular a la población estudiantil a que conceda mayor importancia al hecho de aprender (motivación intrínseca) que a la calificación que obtenga en una materia (motivación extrínseca); igualmente, a que perciba sus habilidades como modificables, que centre su interés más en la experiencia de aprendizaje que en las recompensas externas y que además reconozca la relevancia de las actividades académicas.

\section{Referencias bibliográficas}

Abarca, S. (2001). Psicología de la motivación. San José, Costa Rica: EUNED.

Ajello, A. M. (2003). La motivación para aprender. En C. Pontecorvo (Coord.), Manual de psicología de la educación (pp. 251-271). España: Popular.

Alonso, J. (1992). ¿Qué es lo mejor para motivar a mis alumnos? Madrid: Universidad Autónoma/Instituto de Ciencias de la Educación.
Bandura, A. (1997). Self-efficacy: The exercise of control [Auto-eficacia: $E l$ ejercicio de control]. New York: W. H. Freeman.

Bisquerra, R. (2000). Educación emocionaly bienestar. Barcelona: CISSPRAXIS.

Cerezo, M. T., y Casanova, P. F. (2004). Diferencias de género en la motivación académica de los alumnos de educación secundaria obligatoria. Revista Electrónica de Investigación Psicoeducativa, 2(1), 97-112. Departamento de Psicología, Universidad de Jaén, España. Consultado el 24 de enero de 2008, de: http://www.investigacionpsicopedagogica.org/revista/ articulos/3/espannol/Art_3_31.pdf

de la Herrán Gascón, A. (1999). Didáctica de la motivación. Suplemento Pedagógico Acade, (25), 10-13, y (26), 6-9. Madrid, España. Consultado el 8 de agosto de 2008, de: http:// www.pangea.org/peremarques/dioe/ DIDÁCTICA \% 20DE\%20LA\% 20 MOTIVACIÓN.pdf

García, A. E. (2008). Motivación individual. Consultado el 7 de junio del 2008, de: http://grupos. e magister.com / d ocumento/ administracion_motivacion_y_ organizacion_/1048-38669

Hampton, D., Summer, C., y Webber, R. (1989). Manual de desarrollo de recursos humanos. México: Trillas.

Herrera, F., Ramírez, M. I., Roa, J. M., y Herrera, I. (2004). Tratamiento de las creencias motivacionales en contextos educativos pluriculturales. Revista Iberoamericana de Educación, Sección de Investigación, $\mathrm{N}^{\circ} 37 / 2$. España. Consultado el 5 de 
octubre del 2008, de: http://www. rieoei.org/investigacion/625Herrera. PDF

Naranjo, M. L. (2004). Enfoques conductistas, cognitivos y racional emotivos. San José, C. R.: Universidad de Costa Rica.

Universidad de Sevilla. (2005). Programa Golden5: Una intervención psicoeducativa. Consultado el 14 de abril de 2007, de: http://www.golden5.org/ golden5/

Rice, F. P. (2000). Adolescencia. Desarrollo, relaciones y cultura. Madrid: Prentice Hall.
Santrock, J. (2002). Psicología de la educación. México: Mc Graw-Hill.

Trechera, J. L. (2005). Saber motivar: ¿El palo o la zanahoria? Consultado el 15 de febrero de 2008, de: http://www. psicologia-online.com/articulos/2005/ motivacion.shtml

Valdés, C. (2005). Motivación. Consultado el 12 de noviembre de 2008, de: http://www.gestiopolis.com/canales5/ rrhh/lamotici.htm

Vroom, V. (1964). Work and motivation [Trabajo y motivación]. New York: Wiley. 